\title{
Radiobiologically based treatment plan evaluation for prostate seed implants
}

\author{
Courtney Knaup, BS', Panayiotis Mavroidis, PhD², Carlos Esquivel, MD', Dimos Baltas, MSc, PhD³, Sotirios Stathakis, PhD', \\ Gregory Swanson, MD', Nikos Papanikolaou, PhD' \\ 'Department of Radiation Oncology, University of Texas Health Science Center at San Antonio, San Antonio, TX, USA, ${ }^{2}$ Department of Medical \\ Radiation Physics, Karolinska Institutet \& Stockholm University, Stockholm, Sweden, ${ }^{3}$ Department of Medical Physics \& Engineering, Strahlenklinik, \\ Klinikum Offenbach GmbH, Germany
}

\begin{abstract}
Purpose: Accurate prostate low dose-rate brachytherapy treatment plan evaluation is important for future care decisions. Presently, an evaluation is based on dosimetric quantifiers for the tumor and organs at risk. However, these do not account for effects of varying dose-rate, tumor repopulation and other biological effects. In this work, incorporation of the biological response is used to obtain more clinically relevant treatment plan evaluation.

Material and methods: Eleven patients were evaluated. Each patient received a $145 \mathrm{~Gy}$ implant. Iodine- 125 seeds were used and the treatment plans were created on the Prowess system. Based on CT images the post-implant plan was created. In the post-plan, the tumor, urethra, bladder and rectum were contoured. The biologically effective dose was used to determine the tumor control probability and the normal tissue complication probabilities for the urethra, bladder, rectum and surrounding tissue.

Results: The average tumor control probability and complication probabilities for the urethra, bladder, rectum and surrounding tissue were $99 \%, 29 \%, 0 \%, 12 \%$ and $6 \%$, respectively. These measures provide a simpler means for evaluation and since they include radiobiological factors, they provide more reliable estimation of the treatment outcome.

Conclusions: The goal of this work was to create more clinically relevant prostate seed-implant evaluation by incorporating radiobiological measures. This resulted in a simpler descriptor of treatment plan quality and was consistent with patient outcomes.
\end{abstract}

Key words: LDR, radiobiology, prostate, treatment plan.

\section{Purpose}

Prostate cancer is one of the most common cancers in men, and is responsible for 32000 deaths in the United States in 2010 [1]. Low dose-rate (LDR) brachytherapy is a common radiation therapy treatment used in the management of prostate cancer. The two most common radioactive seeds used in prostate LDR brachytherapy are ${ }^{125} \mathrm{I}$ and ${ }^{103} \mathrm{Pd} .{ }^{125} \mathrm{I}$ has a half-life of 59.4 days and emits a $28 \mathrm{keV}$ gamma ray. Due to its relatively long half-life and penetrating gamma ray, ${ }^{125} \mathrm{I}$ is often preferred for slow growing tumors [2]. The recommended prescription dose for LDR prostate monotherapy is 145 Gy using ${ }^{125}$ I seeds [3]. Post-implant treatment evaluation is a necessary step in LDR prostate brachytherapy. Due to the effects of seed migration and tissue edema, the final position of the seeds may be significantly different from their planned position. Since the seeds emits low energy short range radiation any disturbance in the seed distribution may result in unfavorable hot or cold spots. Thus, accurate post-implant evaluation is important to determine if the implant is satisfactory or if further treatment is needed to achieve the desired outcome.
Currently, the treatment plan evaluation is based on isodose distributions, dose-volume histograms (DVH) as well as mean, maximum and minimum doses in the target and organs at risk (OAR). Most of the evaluation tools currently used in clinical practice are dose-based. These do not account for the radiobiological characteristics of the tumor or OAR that are important in the biological outcome of the therapy. Under some circumstances, plans with similar dose distributions have been shown to have different estimated radiobiological outcomes [4]. Inclusion of radiobiological factors such as tissue radiosensitivity, tissue seriality, treatment time and non-linear response of tissues to radiation may improve the estimation of tumor control and normal tissue complications rates.

In this work, a new 3D treatment plan evaluation tool, based on the radiobiological response of each voxel is used. The goal of this new evaluation method is to simplify post implant evaluation while providing more clinically relevant information. Here, the probability of injury to the tumor and OAR is given as a simple probability. Using spatial information is preferable to the more common DVH-based

\footnotetext{
Address of correspondence: Courtney Knaup, BS, University of Texas Health Science Center San Antonio, Received: 27.05 .11 School of Medicine, Department of Radiation Oncology, 7979 Wurzbach Rd, MC 7889, G260A, San Antonio, Accepted: 20.06 .11 TX 78229, USA, phone: +1316.655.5379, e-mail: knaup@uthscsa.edu

Published: 30.06 .11
} 
radiobiological analysis. Using spatial information, areas of inadequate treatment can be easily visualized and used in creating additional treatment plans that target these areas.

\section{Material and methods}

This study is a retrospective analysis of eleven patients receiving prostate seed implant. Pre-implant prostate volumes were determined using a B-K Medical Leopard 2001 (Mileparken, Denmark) transrectal ultrasound (TRUS) machine with the patients in the lithotomy position. TRUS images were acquired during a continuous sagittal sweep. From the volumetric data acquisition, axial images were reconstructed with $2.5 \mathrm{~mm}$ spacing.

On the axial TRUS images, the prostate and urethra were contoured by a physician. The planning target volume (PTV) was defined to be the prostate volume plus $0.5 \mathrm{~cm}$ margin. The treatment plan was produced using the Prowess Panther 3D Brachy Pro (Prowess, Concord, California, USA). The treatment planning system uses the TRUS images to map out the placement of each seed with respect to a template. The template is simply a metal plate with holes drilled in a regular grid pattern. The template is then used in the operating room to guide each needle to the planned location. The seeds were implanted with precision under TRUS guidance to ensure their placement in the proper locations. All the patients were treated with ${ }^{125} \mathrm{I}$ seeds (BARD, BrachySource model, Covington, GA, USA).

One month after the implant, the patient received a CT scan. Patients were scanned in the supine position and transverse slices were acquired with $2.5 \mathrm{~mm}$ slice thickness. On these CT images the prostate, urethra, bladder and rectum were contoured by a physician. Additionally, the location of each implanted seed was registered using these CT mages. The contoured CT images and seed locations were then used by the in-house software to perform the radiobiological evaluation.

The recommendation given in TG-64 is to perform a post-operative scan 28 days after the implant [5]. The American Brachytherapy Society considers the ideal scan time to be within 4 weeks after the implant [6]. The half-life of prostate edema has been reported to range from 4-25 days, with a mean of 9.3 days $[7,8]$. Scanning several weeks after the procedure allows adequate time for most prostate edema to resolve [6]. This is important for plan evaluation. Due to the use of ${ }^{125}$ I seeds, most of the dose will be delivered after the prostate edema has resolved.

Using the seed positions from the treatment planning system the physical dose distribution was calculated using the TG-43 protocol [9]. Based on the physical dose and type of tissue present in each voxel, the biologically effective dose (BED) and the TCP were determined [4]. The value of the TCP is in part based on calculation of the biologically effective dose (BED), which for the tumor and normal tissues is calculated using equation (1a) and (1b) respectively $[5,10,11]$.

$$
\begin{aligned}
B E D_{\text {tum }}=D_{e f f}\{R B E+ & {\left.\left[\frac{R_{o}}{(\mu+\lambda)(\alpha / \beta)_{t u m}}\right] \times A \times(B-C)\right\}+ } \\
& +\frac{K}{\lambda} \ln \left(\frac{K}{R_{o}}\right)
\end{aligned}
$$

$B E D_{N T}=D_{e f f}\left\{R B E+\frac{R_{o}}{(\mu+\lambda) \times(\alpha / \beta)_{N T}}\right\}$

where,

$$
\begin{aligned}
& A=\frac{1}{1-e^{-\lambda T_{e f f}}} \\
& B=\frac{1-e^{-\lambda T_{e f f}}}{2 \lambda} \\
& C=\frac{1-e^{-T_{e f f}(\mu+\lambda)}}{\mu+\lambda}
\end{aligned}
$$

In equation (1), $R_{0}$ is the initial dose rate and $\lambda$ is the decay constant (for ${ }^{125} \mathrm{I}, \lambda=0.01166$ day $^{-1}$ ). The sublethal damage repair constant $(\mu)$ was calculated using the following expression:

$$
\mu=\frac{\ln (2)}{T_{1 / 2}}
$$

This factor accounts for the decrease in cell kill as the cell repairs damage. Here, a general repair half-life of 15 minutes was assumed for both tumor and normal tissues, making $\mu=2.8$ hour $^{-1}[12,13]$.

The tumor repopulation factor $(K)$ accounts for the growth of new tumor cells during treatment and is calculated as follows:

$$
K=\frac{\ln (2)}{\alpha T_{p o t}}
$$

A potential doubling time $\left(T_{p o t}\right)$ of 42 days was used in this analysis resulting in a repopulation factor $K$ of $0.11 \mathrm{~Gy}$ $\times$ day $^{-1}[12,14]$.

Equation (4) was used for the calculation of the effective dose $\left(D_{\text {eff }}\right)$. The effective treatment time $\left(T_{\text {eff }}\right)$ was determined from equation (5). The endpoint for brachytherapy has been defined as the point where the rate of cell kill is equal to the tumor repopulation factor [15]. For normal tissues it is assumed that $T_{\text {eff }}=\infty$, hence the effective dose is taken to be equal to the total physical dose accumulated over the lifetime of the seeds.

$$
\begin{aligned}
& D_{\text {eff }}=D\left(1-\mathrm{e}^{-\lambda T_{\text {eff }}}\right) \\
& T_{\text {eff }}=\frac{-1}{\lambda} \ln \frac{K}{R o \times R B E}
\end{aligned}
$$

The relative biological effectiveness $(R B E)$ for ${ }^{125} \mathrm{I}$ has been reported to be between 1.4-1.5 [16, 17]. For this study, a middle value of $R B E=1.45$ was used. The specific radiobiological parameters $\alpha / \beta, D_{50}$ and $\gamma$ used for each tissue are shown in Table $1 . D_{50}$ is the dose which gives a $50 \%$ response and $\gamma$ is the maximum normalized dose-response gradient. The $D_{50}$ and $\gamma$ parameters are derived from clinical materials and describe the shape of the dose-response curve [18]. These parameters and the radiobiological model used are similar to those recommended in TG-137 [19]. Biological 
Table 1. Dose-response parameters for each tissue evaluated

\begin{tabular}{llllll} 
Tissue Type & $\boldsymbol{\gamma}$ & $\boldsymbol{\alpha} / \boldsymbol{\beta}$ & $\boldsymbol{D}_{50}$ & $\boldsymbol{s}$ & End point \\
\hline PTV & 4 & 2 & 50 & 1.0 & Control \\
\hline Bladder & 3 & 3 & 80 & 0.3 & Symptomatic Contracture \\
\hline Urethra & 3 & 3 & 230 & 0.01 & RTOG Grade 2 \\
\hline Urethra & 3 & 3 & 275 & 0.01 & RTOG Grade 3 \\
\hline Rectum & 2.2 & 3 & 80 & 0.7 & Proctitis, Fistula, Stenosis
\end{tabular}

parameters are the subject of some uncertainty due to intrapatient radiosensitivity variations [20-23]. The response probability $(P)$ for each voxel was then determined using equation (6) [24-26].

$$
P=\exp (-\exp (\exp (1) \times \gamma-\alpha \times \mathrm{BED}))
$$

The overall response probability for the tumor and normal tissues is calculated using equations (7a) and (7b), respectively.

$$
\begin{aligned}
& P_{\text {tum }}(D, V)=\prod_{i=1}^{N} \mathrm{P}\left(\mathrm{D}_{\mathrm{i}}\right)^{\Delta \mathrm{V}_{\mathrm{i}}} \\
& P_{N T}(D, V)=\left[1-\prod_{i=1}^{N}\left(1-P\left(\mathrm{D}_{\mathrm{i}}, \mathrm{V}_{\mathrm{i}}\right)^{S}\right)^{\Delta \mathrm{V}_{\mathrm{i}}}\right]^{1 / \mathrm{s}}
\end{aligned}
$$

Where $N$ is the total number of voxels in the organ, $s$ is the tissue-specific relative seriality parameter and $\Delta v_{i}$ is the fractional subvolume of the organ irradiated. The overall probability of injury to the involved normal tissues $\left(P_{\mathrm{I}}\right)$ and the complication-free tumor control probability $\left(P_{+}\right)$for the treatment were calculated using equations (8) and (9), respectively. For the calculation of $P_{+}$, both RTOG grade 2 and grade 3 complications to the urethra were considered. The biologically effective uniform dose $(\overline{\bar{D}})$ which is the uniform dose that causes the same tumor control as actual dose distribution for a given treatment was calculated from equation (10).

$$
\begin{aligned}
& P_{1}=1-\prod_{j=l}^{N \text { organs }}\left(1-P_{N T}^{j}\right) \\
& P_{+}=P_{B}-P_{l} \\
& P(\vec{D}) \equiv P(\overline{\bar{D}})
\end{aligned}
$$

The biologically effective uniform dose $(\overline{\bar{D}})$ calculates the uniform dose that would provide the same clinical outcome as the inhomogeneous dose distribution. It is a function of physical dose and tissue specific radiobiological parameters. The general expression of $(\overline{\bar{D}})$ is derived numerically from the first part of the following equation, where for a tissue of uniform radiosensitivity, $(\overline{\bar{D}})$ is given from the analytical formula of the second part of equation (11).

$$
P(\vec{D}) \equiv P(\overline{\bar{D}}) \Rightarrow \overline{\bar{D}}=\frac{e \gamma-\ln (-\ln (P(\vec{D})))}{e \gamma-\ln (\ln 2)}
$$

where $\vec{D}$ denotes the 3-dimensional dose distribution delivered to the tissue and $P(\vec{D})$ is the response probability of the tissue. The second part of the equation has been derived using the Poisson model [4].

\section{Results}

For all of the patients evaluated in this study, the calculated tumor control probability was very high, $99.6 \%$ on average. This result agrees with the dosimetric evaluation. Using the criteria prostate $D_{90}>145$ Gy for a successful implant, all the implants were considered to deliver an adequate dose to the tumor. The results of the radiobiological evaluation are presented in Table 2 . The mean dose to each organ for each patient is given in Table 3. A dosimetric evaluation for each patient is shown in Table 4.

Considering the rectum, Merrick et al. recommend that the maximum dose to the anterior mucosal wall should be less than $120 \%$ of the reference dose [27]. For an implant reference dose of $145 \mathrm{~Gy}$, this $120 \%$ dose is $178 \mathrm{~Gy}$. Comparing the radiobiological results with the dosimetric parameters, patients that had higher rectum complication probabilities tended to have larger maximum rectal doses. However, the patient with the highest maximum rectal dose did not have the highest rectum complication probability. This indicates the weakness of simple dosimetric quantifiers and the advantage of radiobiological evaluation which considers biological factors in relation with the dose distribution. Making an allowance for the bladder, the average complication probability was $0.03 \%$. This is consistent with our clinical experience that the bladder is typically located far enough from the prostate, so that the dose delivered to it is low.

Considering the urethra, the average probability for RTOG grade 2 and grade 3 complications was $38.95 \%$ and $9.81 \%$, respectively [28]. There are several dosimetric quantifiers reported in the literature to predict urethral complications, two of which are presented in Table 4 [29-31]. Wallner et al. have shown that maximum urethral doses of 447 Gy and 592 Gy are related to RTOG grade 0-1 and grade $2-3$ complications, respectively $[32,33]$. However, it is seen in Table 4 that for our group of patients these dose quantifiers provide conflicting results.

Since the radiobiological evaluation in this system is calculated using spatial dose information, is it possible to 
Table 2. Results of radiobiological evaluation. The $P_{+} G 2$ and $P_{+} G 3$ columns give the $P_{+}$calculation for urethra RTOG grade 2 and 3 injury, respectively

\begin{tabular}{ccccccccc} 
Patient & TCP (\%) & $\begin{array}{c}\text { NTCP } \\
\text { Bladder (\%) }\end{array}$ & $\begin{array}{c}\text { NTCP } \\
\text { Rectum (\%) }\end{array}$ & $\begin{array}{c}\text { NTCP } \\
\text { Urethra G2 (\%) }\end{array}$ & $\begin{array}{c}\text { NTCP } \\
\text { Other (\%) }\end{array}$ & $\begin{array}{c}P_{+} \text {G2 } \\
(\%)\end{array}$ & $\begin{array}{c}\text { NTCP } \\
\text { Urethra G3 (\%) }\end{array}$ & $\begin{array}{c}P_{+} \text {G3 } \\
(\%)\end{array}$ \\
\hline 1 & 99.8 & 0.2 & 2.2 & 0.1 & 7.3 & 90.3 & 0.0 & 90.3 \\
\hline 2 & 100.0 & 0.0 & 6.9 & 20.1 & 11.6 & 65.8 & 0.4 & 82.0 \\
\hline 3 & 99.5 & 0.0 & 8.5 & 2.3 & 8.1 & 81.7 & 0.0 & 83.6 \\
\hline 4 & 99.1 & 0.0 & 21.4 & 2.2 & 6.4 & 71.1 & 0.0 & 72.8 \\
\hline 5 & 99.9 & 0.0 & 7.7 & 30.3 & 7.8 & 59.3 & 1.4 & 83.9 \\
\hline 6 & 99.8 & 0.0 & 16.3 & 27.7 & 3.9 & 58.0 & 1.0 & 79.5 \\
\hline 7 & 99.9 & 0.0 & 6.0 & 37.4 & 1.5 & 57.9 & 3.1 & 89.7 \\
\hline 8 & 99.8 & 0.0 & 6.5 & 66.6 & 4.3 & 29.7 & 17.4 & 73.7 \\
\hline 9 & 100.0 & 0.0 & 13.7 & 19.9 & 3.4 & 66.9 & 0.0 & 83.4 \\
\hline 10 & 100.0 & 0.0 & 13.3 & 28.3 & 8.5 & 56.9 & 1.0 & 78.5 \\
\hline 11 & 99.9 & 0.0 & 25.5 & 85.0 & 6.3 & 10.4 & 48.2 & 36.1 \\
\hline Avg \pm SD & $99.8 \pm 0.3$ & $0.0 \pm 0.0$ & $11.6 \pm 7.1$ & $29.1 \pm 26.6$ & $6.3 \pm 2.8$ & $58.9 \pm 22.3$ & $5.0 \pm 10.0$ & $77.6 \pm 14.9$
\end{tabular}

Table 3. Mean dose to each organ for each patient

\begin{tabular}{ccccc} 
Patient & Prostate (Gy) & Urethra (Gy) & Bladder (Gy) & Rectum (Gy) \\
\hline 1 & 309 & 182 & 25.4 & 29.0 \\
\hline 2 & 343 & 241 & 25.2 & 42.1 \\
\hline 3 & 342 & 213 & 39.4 & 34.0 \\
\hline 4 & 319 & 220 & 14.9 & 51.6 \\
\hline 5 & 335 & 259 & 27.4 & 36.7 \\
\hline 6 & 300 & 247 & 18.6 & 49.6 \\
\hline 7 & 297 & 261 & 15.4 & 33.3 \\
\hline 8 & 348 & 287 & 15.5 & 34.0 \\
\hline 9 & 321 & 242 & 19.6 & 29.9 \\
\hline 10 & 353 & 225 & 17.2 & 32.7 \\
\hline 11 & 357 & 320 & 18.0 & 50.9 \\
\hline Avg \pm SD & $329 \pm 21$ & $245 \pm 37$ & $21.5 \pm 7.4$ & $38.5 \pm 8.5$
\end{tabular}

Table 4. Dosimetric evaluation based on the $D_{90}$ and $V_{150}$ from the prostate and maximum rectal dose. The bracketed organ indicated the target for each dose quantifier

\begin{tabular}{|c|c|c|c|c|c|c|c|}
\hline Patient & $\begin{array}{c}\text { PTV } \\
D_{90} \text { (Gy) }\end{array}$ & $\begin{array}{c}\text { PTV } \\
V_{150}(\%)\end{array}$ & $\begin{array}{c}\text { Rectum } \\
D_{\max }(G y) \\
\end{array}$ & $\begin{array}{c}\mathrm{D}_{90}>145 \mathrm{~Gy} \\
\text { [tumor] }\end{array}$ & $\begin{array}{c}\mathrm{D}_{90}<180 \mathrm{~Gy} \\
\text { [urethra] }\end{array}$ & $\begin{array}{c}\mathrm{V}_{150}<60 \% \\
\text { [urethra] }\end{array}$ & $\begin{array}{c}\mathrm{RD}_{\max }<178 \text { Gy } \\
\text { [rectum] }\end{array}$ \\
\hline 1 & 164 & 63.9 & 135.5 & yes & yes & no & yes \\
\hline 2 & 184 & 78.4 & 151 & yes & no & no & yes \\
\hline 3 & 161 & 71.8 & 183 & yes & yes & no & no \\
\hline 4 & 160 & 71.4 & 231 & yes & yes & no & no \\
\hline 5 & 184 & 78.4 & 165 & yes & no & no & yes \\
\hline 6 & 169 & 72.2 & 174 & yes & yes & no & yes \\
\hline 7 & 154 & 61.9 & 155 & yes & yes & no & yes \\
\hline 8 & 171 & 77.6 & 161 & yes & yes & no & yes \\
\hline 9 & 180 & 76 & 304 & yes & no & no & no \\
\hline 10 & 188 & 78.4 & 213 & yes & no & no & no \\
\hline 11 & 190 & 82.3 & 210 & yes & no & no & no \\
\hline
\end{tabular}



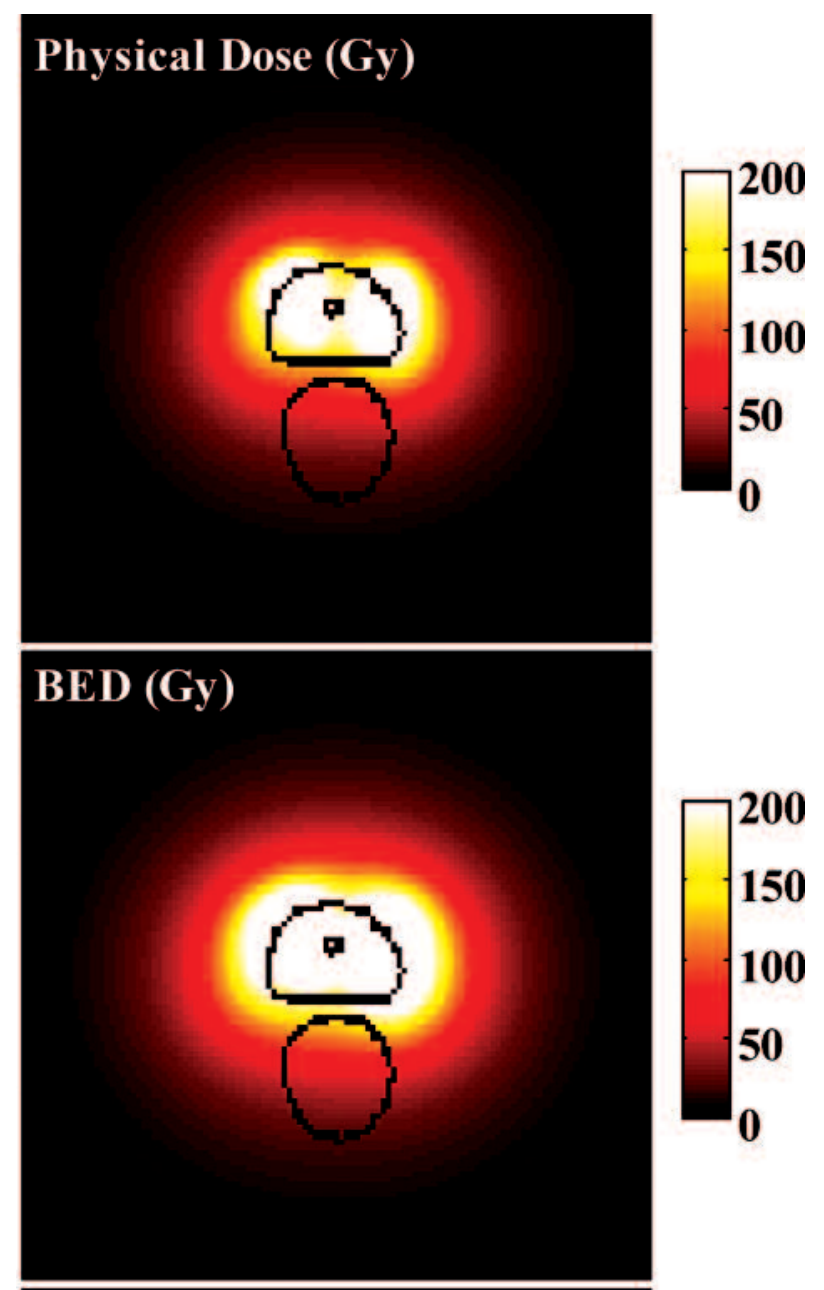

\section{Response Probability (\%)}
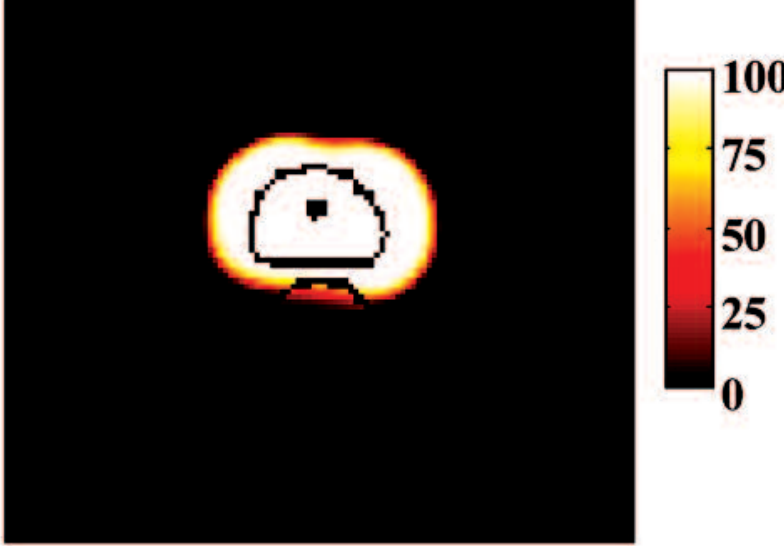

Fig. 1. Images of the physical dose, BED and response probability for patient 3 . Organ contours are overlaid on each image

visualize the response probabilities as one might do with physical dose. More simplistic radiobiological analyses that are based on DVH information do not have this capability. Figures 1 and 2 show that the response probability distribution is different from the dose distribution. This illustrates the advantage of incorporating radiobiological
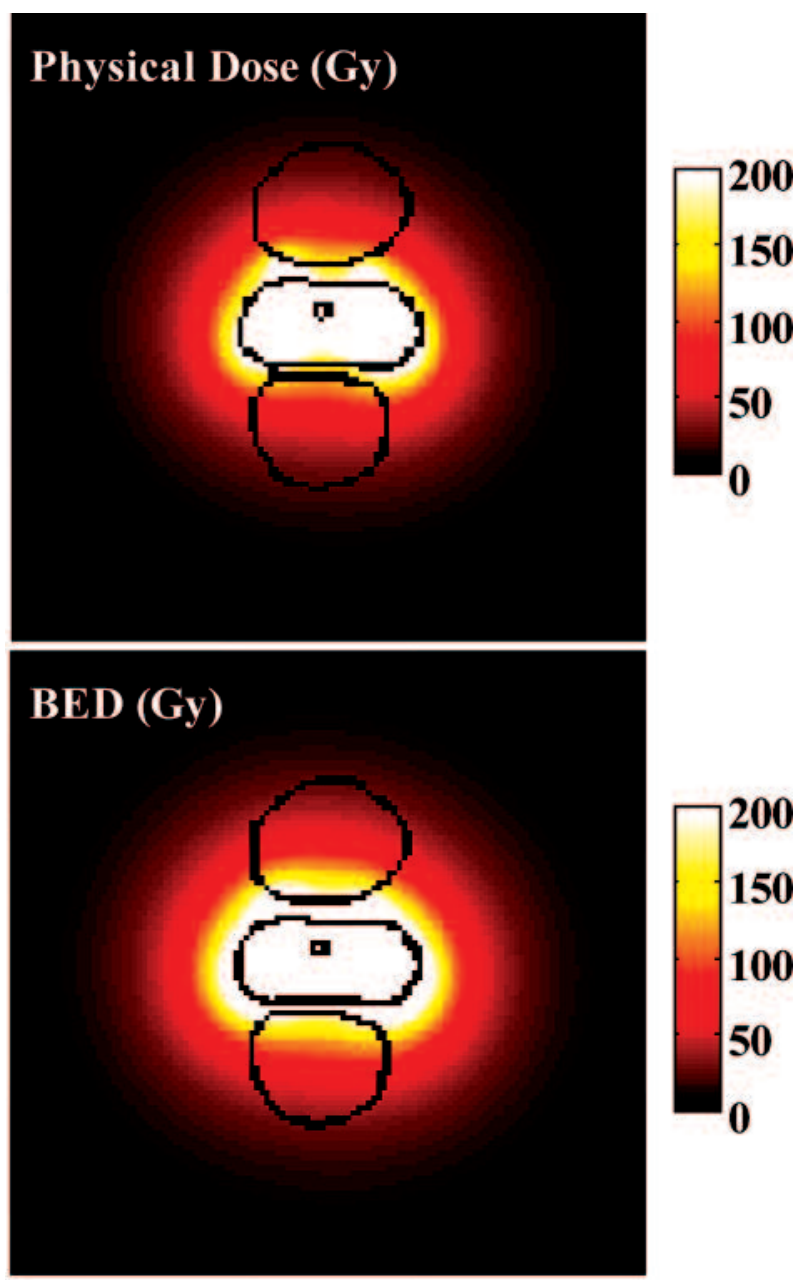

\section{Response Probability (\%)}

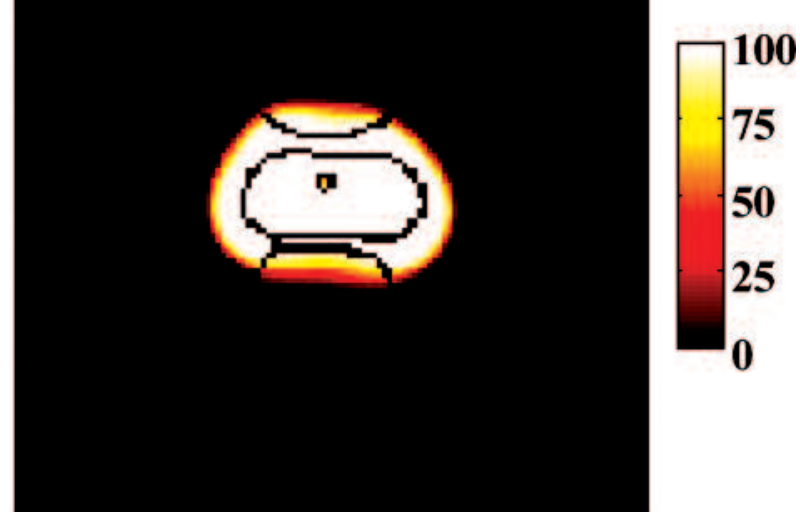

Fig. 2. Images of the physical dose, BED and response probability for patient 11 . Organ contours are overlaid on each image

considerations to the implant evaluation, since it provides better estimation of the clinical outcome compared to physical dose alone.

The upper images in Figs. 3 and 4 show that patient 3 represents more conformal treatment, while patient 11 represents a less conformal treatment. The DVH of patient 11 

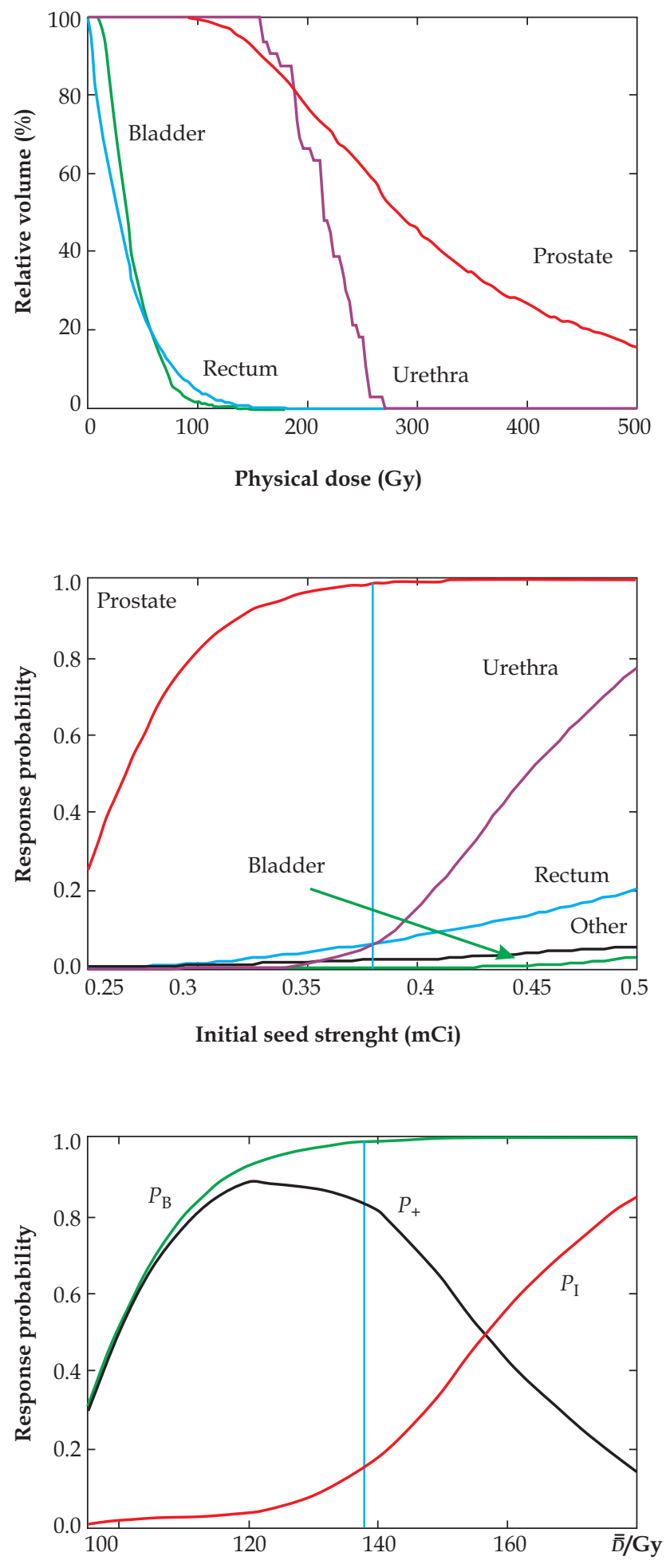

Fig. 3. The plots shown above are for patient 3 , calculated with urethra RTOG grade 2 injury. The upper image is the DVH. The middle image shows the response probabilities of each organ for an identical implant, but with different initial seed activity. The lower figure shows the $P_{+}, P_{B}$ and $P_{I}$ vs. $\overline{\bar{D}}$ curves for identical implants with different initial seed activities. The vertical line indicates the clinically prescribed seed activity (middle) or $\overline{\bar{D}}$ (lower) shows that the dose distributions to the rectum and urethra are significantly higher than those of patient 3 , while the dose distributions to the tumor are comparable. The initial seed activities for patients 3 and 11 were 0.38 and $0.37 \mathrm{mCi}$, respectively. The middle plot of Fig. 3 indicates that the $P_{I}$ begins to rise just above this activity. The middle plot of Fig. 4 shows that even for low initial seed activities the $P_{B}$ is saturated and $P_{I}$ is significant. This would seem to indicate that too many seeds were used in this implant. The lower plot of Fig. 3 shows that $P_{+}$is high for actual implanted $\overline{\bar{D}}$ of $138 \mathrm{~Gy}$. However, this plot also indicates that the most ideal balance of $P_{B}$ and $P_{I}$ is obtained at a equal to $120 \mathrm{~Gy}$. The lower plot of Fig. 4 shows that the $\overline{\bar{D}}$ which would be delivered with even low strength seeds is significantly higher than the ideal level. Figures 5 and 6 include the response of the urethra to RTOG grade 3 injury. The higher dose limit for grade 3 injury has the effect of pushing the $P_{I}$ curve to higher doses, thus broadening the $P_{+}$curve.

\section{Discussion}

The evaluation of prostate implants is commonly done using dose quantifiers such as $D_{100}, D_{90}$ and $V_{150}$ to compare the implant to known TCP and NTCP. However, such simple quantifiers demonstrate only a small piece of the picture. Prostate implants with similar $D_{90}$ can have significantly different $D_{50}$ and $D_{\max }$. To glean a better idea of the TCP and NTCP one must consider many dose quantifiers. At some point, the increased information that should provide the basis for an excellent evaluation becomes overwhelming. With the proposed methodology based on the spatial distribution of the probability of response, the effectiveness of a given dose distribution is much more clear, easy to assess and clinically relevant.

Figures 2 and 3 presents that the response probability distribution eliminates much of the visual noise due to low doses that may not have a clinical effect. These images may be used to indicate regions that might need further irradiation. Radiobiological evaluation of a treatment provides additional information about the fitness of the implant and a closer association to the clinical outcome. This evaluation takes into account the dose-response characteristics of the tumor and normal tissues that are important in the treatment. The concurrent display of both the radiobiological evaluation and the dosimetric data shows their complementary relation in analyzing the treatment.

The TCP for all of the patients was very high. This result is consistent with the dosimetric data, which shows that all the patients had prostate $D_{90}$ well above the acceptable criteria of $145 \mathrm{~Gy}$. Sparing the urethra represents a technical difficulty since the urethra passes directly through the prostate. Thus, one must balance the need for adequate prostate dose coverage while minimizing the urethral dose. Even using dose limiting techniques, the risks of urethra complications was high for several patients. Radiobiological parameters for the urethra are sparsely reported in the literature. For this study, the parameters were chosen such that the calculated response probability was consistent with dose-response information reported by others. Takeda et al. has reported that for a patient cohort with average urethral 

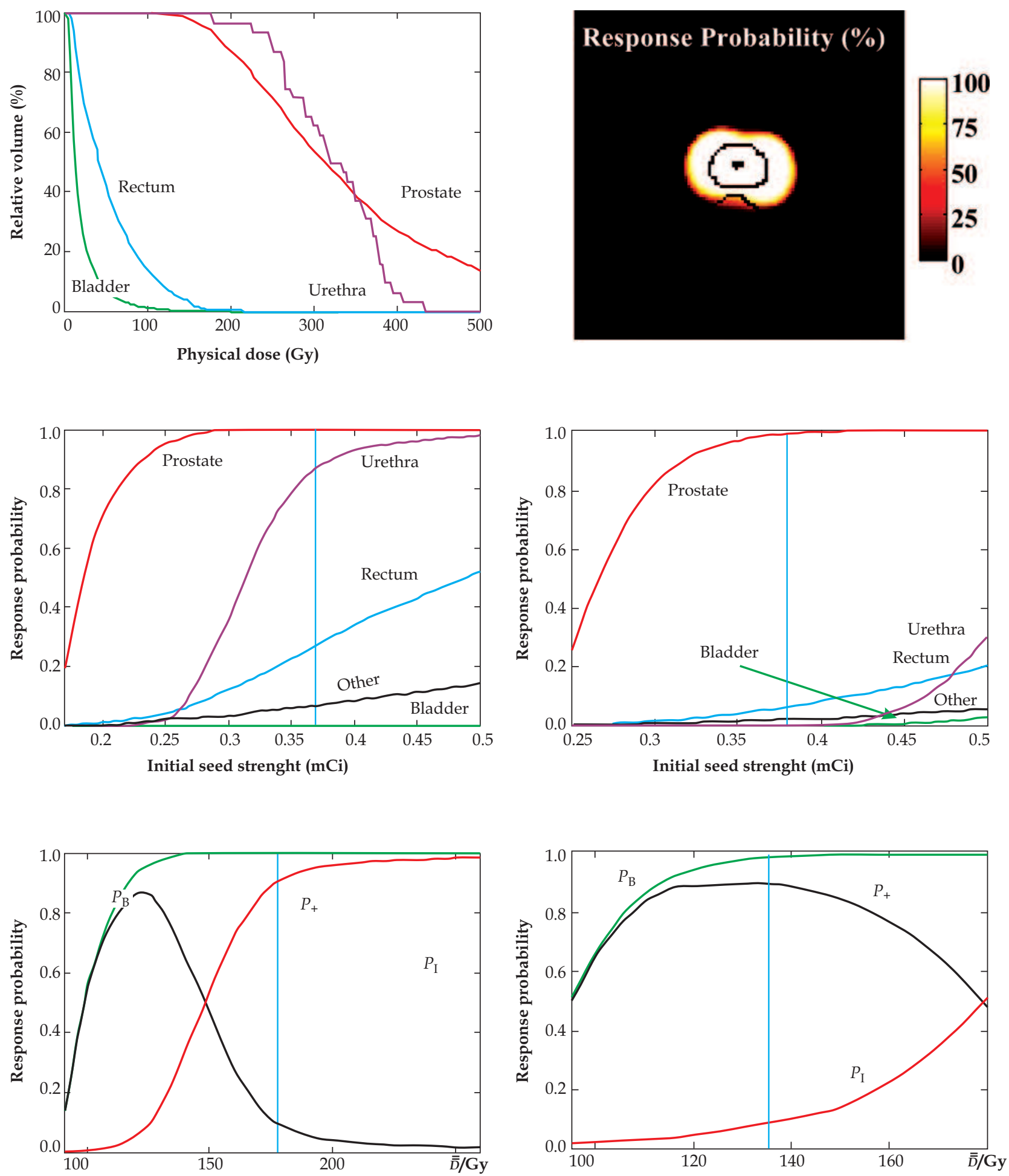

Fig. 4. The plots presented above are for patient 11 , calculated with urethra RTOG grade 2 injury. The upper image is the DVH. The middle image shows the response probabilities of each organ for an identical implant but with different initial seed activity. The lower figure shows the $P_{+}$, $P_{B}$ and $P_{I}$ vs. $\overline{\bar{D}}$ curves for identical implants with different initial seed activities. The vertical line indicates the clinically prescribed seed activity (middle) or $\overline{\bar{D}}$ (lower)

Fig. 5. The plots shown above are for patient 3, calculated with urethra RTOG grade 3 injury. The upper image shows the spatial response probability distribution. The middle image shows the response probabilities of each organ for an identical implant but with different initial seed activity. The lower figure shows the $P_{+}, P_{B}$ and $P_{I}$ vs. $\overline{\bar{D}}$ curves for identical implants with different initial seed activities. The vertical line indicates the clinically prescribed seed activity (middle) or $\overline{\bar{D}}$ (lower) 

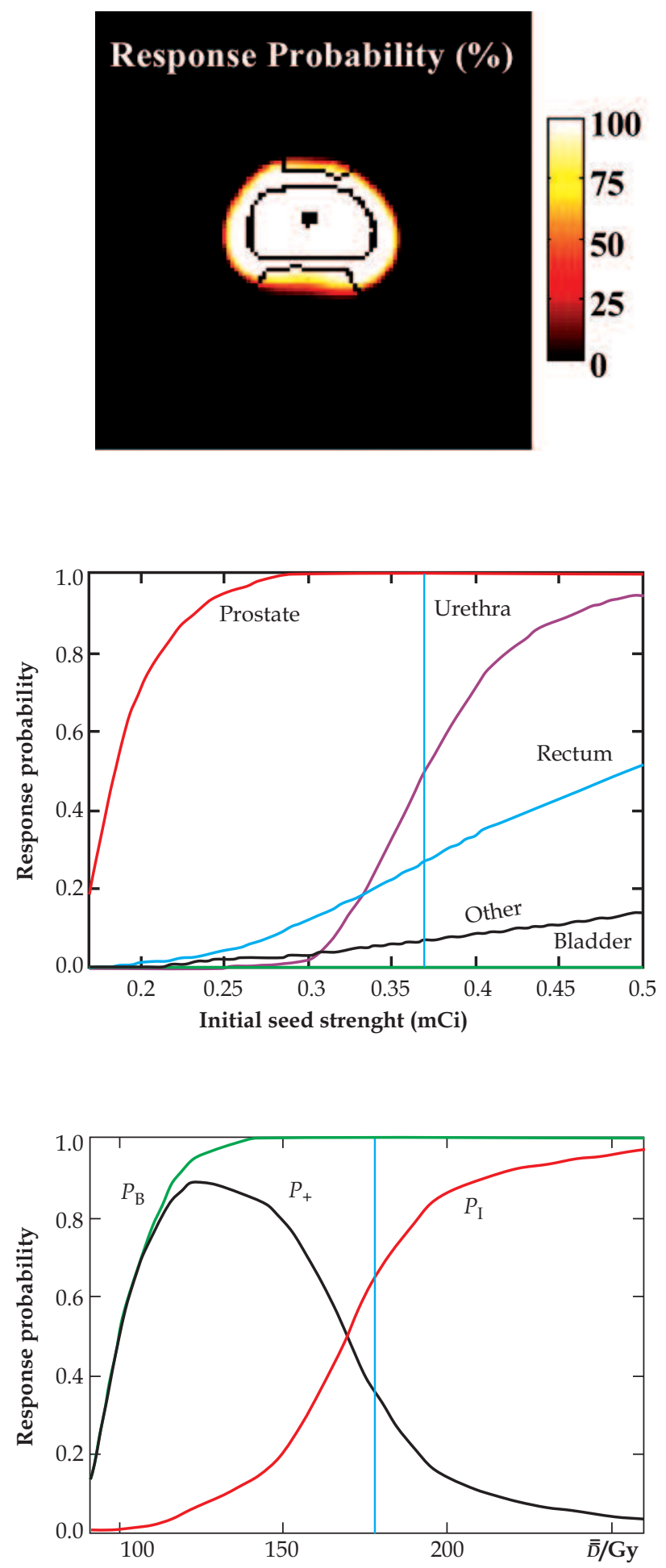

Fig. 6. The plots presented above are for patient 11, calculated with urethra RTOG grade 3 injury. The upper image shows the spatial response probability distribution. The middle image shows the response probabilities of each organ for an identical implant but with different initial seed activity. The lower figure shows the $P_{+}, P_{B}$ and $P_{I}$ vs. $\overline{\bar{D}}$ curves for identical implants with different initial seed activities. The vertical line indicates the clinically prescribed seed activity (middle) and $\overline{\bar{D}}$ (lower)
$D_{90}=150$ Gy, $90 \%$ experienced RTOG grade 2 symptoms and $6.5 \%$ experienced grade 3 symptoms [34]. Additionally, Thomas et al. has reported that for a patient cohort with average urethral $D_{90}=105 \mathrm{~Gy}, 36 \%$ and $7 \%$ experience grade 2 and 3 symptoms, respectively [35]. Thus, the urethra complication rates predicted for the patients in this study are comparable with these results.

Comparing the radiobiological results with the dosimetric parameters, patients that had higher rectum complication probabilities tended to have larger maximum rectal doses. However, the patient with the highest maximum rectal dose did not have the highest rectum complication probability. Authors have reported that rectal complications are most frequent eight months post-implant, with $8-18 \%$ of patients reporting complications $[27,36]$. The predicted rectal complication rate calculated for our patient cohort was similar, at $11 \%$. Combining all the tissue-response information, the success of the treatment may be given as a single value, the complication-free tumor control probability $\left(P_{+}\right)$. The average $P_{+}$for the patient cohort was $58.9 \%$. However, the value varied amongst the patients, from $10.4 \%$ to $90.3 \%$.

Two specific patients were selected in order to present the results of a conformal implant and a less conformal implant. Comparing Figs. 1 and 2, the physical dose distribution for patient 3 seems uniform compared to patient 11 which is characterized by higher inhomogeneities in the dose distribution. The response probability distribution shows that the tumor response for both patients is high. It also shows that while the physical dose distributions look quite different, the tumor responses are similar. However, the response probability distribution for patient 11 shows that the urethra response is high and that a greater area of normal tissue surrounding the tumor is responding compared with patient 3 . Figure 3 shows that for patient 3 the implant was ideal with respect to the initial seed strength and overall $\overline{\bar{D}}$ to the tumor. Figure 4 indicates that an ideal treatment would require much lower activity seeds for patient 11 . This would seem to indicate that too many seeds were implanted in this patient for the given source strength. Figures 5 and 6 represent the evaluation where RTOG grade 3 injury is considered as the urethra endpoint of interest. Since the urethra is the most significant component of $P_{I}$, the higher dose-response limit pushes the $P_{I}$ curve to higher doses. This has the effect of broadening the $P_{+}$curve toward higher doses.

The clinical outcomes for the patient cohort were consistent with our predicted outcomes. All patients had PSA scores and digital rectal exams indicating satisfactory improvement. Most patients with low normal tissue response probabilities, including patient 3 , reported minimal symptoms such as increased nocturia and slight rectal irritation. Patients with higher response probabilities reported more severe symptoms such as erectile dysfunction, bloody stools, increased nocturia, urinary hesitancy and pain with urination. Patient 11 reported the most severe symptoms of the patient cohort.

The main advantage of this evaluation method is that it is based on the response probability calculated from each voxel. This allows for response probability maps to be generated. The major limitation of this system stems from the use of specific radiobiological parameters, which are 
known to be the subject of various types of uncertainties. Thus, the probabilities calculated should only be considered as estimates of the response for a typical patient. However, this limitation is also shared by all the common radiobiological and dosimetric quantifiers, because the degree of radiation induced effect for a specified dose level is also variable within a patient population.

\section{Conclusions}

The goal of this study was to improve the evaluation of prostate seed implants by developing a radiobiological evaluation system based on dose-response probabilities. The system proposed here uses the spatial dose information from the treatment planning system combined with tissue-specific radiobiological factors to estimate tumor control probability and normal tissue complication probabilities for each implant. The evaluation of a small patient cohort with both the proposed methodology and common dosimetric measures shows that the proposed system yields results consistent with those of reported patient outcomes. In summary, this system uses a potentially more comprehensive radiobiological analysis to estimate patient outcomes and the results are reported in a simple and intuitive manner. The authors envision that such a system may be used along with traditional dose quantifiers to provide more inclusive and clinically relevant evaluation of prostate seed implants.

\section{References}

1. http://www.cancer.org/Cancer/ProstateCancer/Overview Guide/prostate-cancer-overview-key-statistics.

2. Ling CC. Permanent implants using Au-198, Pd-103 and I-125: radiobiological considerations based on the linear quadratic model. Int J Radiat Oncol Biol Phys 1992; 23: 81-87.

3. Nag S, Beyer D, Friedland J et al. American Brachytherapy Society (ABS) recommendations for transperineal permanen brachytherapy of prostate cancer. Int J Radiat Oncol Biol Phys 1999; 44: 789-799.

4. Mavroidis P, Lind BK, Brahme A. Biologically effective uniform dose (D) for specification, report and comparison of dose response relations and treatment plans. Phys Med Biol 2001; 46: 2607-2630.

5. Yu Y, Anderson LL, Li Z et al. Permanent prostate seed implant brachytherapy: report of the American Association of Physicists in Medicine Task Group No. 64. Med Phys 1999; 26: 2054-2076.

6. Nag S, Bice W, DeWyngaert K et al. The American Brachytherapy Society recommendations for permanent prostate brachytherapy postimplant dosimetric analysis. Int J Radiat Oncol Biol Phys 2000; 46: 221-230.

7. Waterman FM, Yue N, Corn BW et al. Edema associated with I-125 or Pd-103 prostate brachytherapy and its impact on postimplant dosimetry: an analysis based on serial CT acquisition. Int J Radiat Oncol Biol Phys 1998; 41: 1069-1077.

8. Crook J, McLean M, Yeung I et al. MRI-CT fusion to assess postbrachytherapy prostate volume and the effects of prolonged edema on dosimetry following transperineal interstitial permanent prostate brachytherapy. Brachytherapy 2004; 3: 55-60.

9. Nath R, Anderson LL, Luxton G et al. Dosimetry of interstitial brachytherapy sources: recommendations of the AAPM Radiation Therapy Committee Task Group No. 43. American
Association of Physicists in Medicine. Med Phys 1995; 22: 209-234.

10. Zaider M, Hanin L. Biologically-equivalent dose and long-term survival time in radiation treatments. Phys Med Biol 2007; 52: 6355-6362.

11. Kim Y, Tome WA. Risk-adaptive optimization: selective boosting of high-risk tumor subvolumes. Int J Radiat Oncol Biol Phys 2006; 66: 1528-1542.

12. Wang JZ, Guerrero M, Li XA. How low is the alpha/beta ratio for prostate cancer? Int J Radiat Oncol Biol Phys 2003; 55: 194-203.

13. Brenner DJ, Hall EJ. Conditions for the equivalence of continuous to pulsed low dose rate brachytherapy. Int J Radiat Oncol Biol Phys 1991; 20: 181-190.

14. Armpilia CI, Dale RG, Coles IP et al. The determination of radiobiologically optimized half-lives for radionuclides used in permanent brachytherapy implants. Int J Radiat Oncol Biol Phys 2003; 55: 378-385.

15. Antipas V, Dale RG, Coles IP. A theoretical investigation into the role of tumour radiosensitivity, clonogen repopulation, tumour shrinkage and radionuclide RBE in permanent brachytherapy implants of 125I and 103Pd. Phys Med Biol 2001; 46: 2557-2569.

16. Wuu CS, Zaider M. A calculation of the relative biological effectiveness of 125I and 103Pd brachytherapy sources using the concept of proximity function. Med Phys 1998; 25: 2186-2189.

17. Ling CC, Li WX, Anderson LL. The relative biological effectiveness of I-125 and Pd-103. Int J Radiat Oncol Biol Phys 1995; 32: 373-378.

18. Kallman P, Agren A, Brahme A. Tumour and normal tissue responses to fractionated non-uniform dose delivery. Int I Radiat Biol 1992; 62: 249-262.

19. Nath R, Bice WS, Butler WM et al. AAPM recommendations on dose prescription and reporting methods for permanent interstitial brachytherapy for prostate cancer: report of Task Group 137. Med Phys 2009; 36: 5310-5322.

20. Mavroidis P, Ferreira BC, Shi C et al. Treatment plan comparison between helical tomotherapy and MLC-based IMRT using radiobiological measures. Phys Med Biol 2007; 52: 3817-3836.

21. Ferreira BC, Mavroidis P, Adamus-Gorka M et al. The impact of different dose-response parameters on biologically optimized IMRT in breast cancer. Phys Med Biol 2008; 53: 2733-2752.

22. Mavroidis P, Laurell G, Kraepelien T et al. Determination and clinical verification of dose-response parameters for esophageal stricture from head and neck radiotherapy. Acta Oncol 2003; 42: 865-881.

23. Mavroidis P, al-Abany M, Helgason AR et al. Dose-response relations for anal sphincter regarding fecal leakage and blood or phlegm in stools after radiotherapy for prostate cancer. Radiobiological study of 65 consecutive patients. Strahlenther Onkol 2005; 181: 293-306.

24. Strigari L, Orlandini LC, Andriani I et al. A mathematical approach for evaluating the influence of dose heterogeneity on TCP for prostate cancer brachytherapy treatment. Phys Med Biol 2008; 53: 5045-5059.

25. Li XA, Wang JZ, Stewart RD et al. Dose escalation in permanent brachytherapy for prostate cancer: dosimetric and biological considerations. Phys Med Biol 2003; 48: 2753-2765.

26. Haworth A, Ebert M, Waterhouse D et al. Assessment of i-125 prostate implants by tumor bioeffect. Int J Radiat Oncol Biol Phys 2004; 59: 1405-1413.

27. Merrick GS, Butler WM, Dorsey AT et al. Rectal dosimetric analysis following prostate brachytherapy. Int J Radiat Oncol Biol Phys 1999; 43: 1021-1027.

28. Lawton CA, Won M, Pilepich MV et al. Long-term treatment sequel following external beam irradiation for adenocarcino- 
ma of the prostate: analysis of RTOG studies 7506 and 7706 Int J Radiat Oncol Biol Phys 1991; 21: 935-939.

29. Stock RG, Stone NN. Importance of post-implant dosimetry in permanent prostate brachytherapy. Eur Urol 2002; 41: 434-439.

30. Stone NN, Stock RG. Complications following permanent prostate brachytherapy. Eur Urol 2002; 41: 427-433.

31. Merrick GS, Butler WM, Tollenaar BG et al. The dosimetry of prostate brachytherapy-induced urethral strictures. Int J Radiat Oncol Biol Phys 2002; 52: 461-468.

32. Wallner K, Roy J, Harrison L. Dosimetry guidelines to minimize urethral and rectal morbidity following transperineal I-125 prostate brachytherapy. Int J Radiat Oncol Biol Phys 1995; 32: $465-471$.

33. Wallner K, Merrick G, True L et al. I-125 versus Pd-103 for low-risk prostate cancer: morbidity outcomes from a prospective randomized multicenter trial. Cancer J 2002; 8: 67-73.

34. Takeda K, Jingu K, Koto M et al. Predicting the severity of acute urinary toxicity after brachytherapy with iodine- 125 for localized prostate cancer. Tohoku J Exp Med 2011; 223: 55-60.

35. Thomas C, Keyes M, Liu M et al. Segmental urethral dosimetry and urinary toxicity in patients with no urinary symptoms before permanent prostate brachytherapy. Int J Radiat Oncol Biol Phys 2008; 72: 447-455.

36. Gelblum DY, Potters L. Rectal complications associated with transperineal interstitial brachytherapy for prostate cancer. Int J Radiat Oncol Biol Phys 2000; 48: 119-124. 